La vida al otro lado de la frontera: estudio

fenomenológico del proceso de aculturación de las mujeres hispanas en los Estados Unidos

Arturo Enrique Orozco Vargas

EL GUSTO POR LA LECTURA Y EL CINE COMO INDICADOR DE LA RECONFORMACIÓN DE LAS SUBJETIVIDADES FEMENINAS: LAS ADOLESCENTES EN JALISCO

GLORIA BRICEÑO ALCARAZ

De la ideología a la identidad en mujeres con cáncer cervicouterino

Ma. del Carmen Calderón Benavides 


\title{
La vida al otro lado de la frontera: estudio fenomenológico del proceso de aculturación de las mujeres hispanas en los Estados Unidos
}

\section{Arturo Enrique Orozco Vargas*}

\author{
* University of North Texas. País: \\ E.U.A. Correo electrónico: \\ arturoorozco@my.unt.edu
}

RECEPCIÓN: 6 DE JUNIO DE 2013/ ACEPTACIÓN: 18 DE MARZO DE 2015

\section{Resumen}

Los obstáculos encontrados en el camino hacia los Estados Unidos y la adaptación a las normas y costumbres norteamericanas representan un enorme desafío para las inmigrantes hispanas. El objetivo de este estudio cualitativo fue investigar desde un enfoque fenomenológico el significado de las experiencias de vida de ocho mujeres de origen hispano quienes residen en el Metroplex de Dallas, Texas. Para lograr dicho cometido, se diseñó un protocolo de entrevista semi-estructurado cuya finalidad fue explorar los motivos que las llevaron a dejar sus países de origen y describir las principales experiencias que han vivido en su propio proceso de aculturación. En la narrativa de las participantes se identificaron factores fuertemente relacionados con este proceso entre los que destacaron: la búsqueda de mejores condiciones económicas en los Estados Unidos, los sentimientos de nostalgia y tristeza, el miedo a ser deportadas, la adaptación a la cultura norteamericana, el hecho de que sus hijos son bilingües, el continuo apoyo de su familia y amigos, la posibilidad de trabajar en la fuerza laboral, la barrera del idioma inglés y 
el contacto con otros latinos. Como quedó constatado en este estudio, la presencia de estos factores es un elemento clave para entender las vicisitudes que conlleva la vida de las inmigrantes de origen hispano una vez que logran cruzar la frontera.

Palabras clave: aculturación, desafíos, oportunidades, recursos, fenomenología.

\section{Abstract}

The obstacles found on the route towards the United States and the adaptation to the American norms and customs entails a big challenge for Hispanic immigrants. The objective of this qualitative study was to investigate based on a phenomenological approach the meaning of the life experiences of eight women of Hispanic origin who are living in the Dallas metroplex in Texas. In order to achieve this goal, a semi-structured interview protocol was designed whose purpose was to explore the motives they had to leave their countries of origin and describe the main experiences they have lived in their own process of acculturation. In the participants experiences, specific factors strongly related to this process were identified, such as leaving their native countries in search of better economic conditions in the United States, feelings of nostalgia and sadness, the fear of being deported, the adaptation to the American culture, their children's bilingualism, the continuous support of their family and friends, the possibility of working on the workforce, the barrier of English language and contact with other Latinos. As it was confirmed in this study, the presence of these factors is a key element for 
understanding the vicissitudes associated with the life of immigrants of Hispanic origin once they cross the border.

Keywords: acculturation, challenges, opportunities, assets, phenomenology.

\section{Introducción}

La inmigración es un fenómeno social, económico, político, y cultural presente en todos los países alrededor del mundo, el cual se caracteriza principalmente por la búsqueda de mejores condiciones de vida. En él convergen los valores, motivaciones, aspiraciones y sueños de las mujeres y hombres que deciden emprender una larga travesía hacia una nueva sociedad con los múltiples desafíos que esta experiencia conlleva (Boneva y Frieze, 2001). La decisión de emigrar puede involucrar a una sola persona, un grupo de personas o los miembros de una familia quienes deciden moverse a un nuevo país en el cual probablemente encontrarán una cultura distinta, un idioma diferente y muchas otras condiciones sociales, económicas y políticas que los inmigrantes no conocieron en sus países de origen.

Aunque existen un sinfín de razones por las cuales familias o grupos de personas sin parentesco familiar entre ellos deciden emigrar a otro país, el principal motivo sigue siendo la búsqueda de mejores condiciones económicas (Bacallao y Smokowski, 2007; González, 2002; Musetescu, 2006; Sánchez-Quintanar, 2005). Otros 
factores que explican el fenómeno de la inmigración alrededor del mundo incluyen el deseo de reintegrarse con aquellos miembros de la familia quienes se encuentran viviendo en otro país, la búsqueda de mejores oportunidades educativas y lugares para desarrollar sus carreras profesionales, y el acceso a servicios de salud más completos. De manera particular, durante las dos últimas décadas, cada vez son más las personas que también buscan emigrar a otros países como una forma de escapar de los secuestros, las amenazas políticas y los ataques relacionados con el tráfico de drogas (Aisenstein, 2001; Andreas, 2000; Bourhis, Moise, Perreault, y Senecal, 1997; Hämäläinen, 2002; Hernandez y McGoldrick, 1999; Iñiguez, 2008; Sánchez-Huesca y Arellanez-Hernández, 2011; Zúñiga, Leite y Nava, 2004).

\section{El proceso de aculturación}

Debido al enorme impacto que representa la inmigración, para la mayoría de las inmigrantes hispanas ${ }^{1}$, la decisión de dejar sus países

${ }^{1}$ Los términos "hispano" y "latino" han sido incluidos en la literatura científica para describir a los inmigrantes provenientes de cualquier país latinoamericano quienes se encuentran viviendo en los Estados Unidos. Aunque el término hispano fue propuesto y es utilizado por organismos federales norteamericanos en un sinfín de documentos gubernamentales, un gran número de autores prefieren y emplea el término latino. En la presente investigación, ambos términos serán usados para describir a esta población. de origen y adaptarse a una nueva cultura ha transformado radicalmente sus vidas (Gamio, 1969). En este proceso lleno de obstáculos y penurias, las mujeres inmigrantes tienen que emplear un sinnúmero de estrategias con la finalidad de ajustar su forma de pensar y comportarse a las nuevas circunstancias presentes en el país de residencia. Diferentes autores han usado el término aculturación para 
describir esta adaptación la cual se caracteriza por la transformación paulatina del estilo de vida de los inmigrantes y sus familias (Melville, 1978; Adelman, 1988; George y Ramkissoon, 1998; Shin, 1999; Yeh et al., 2003; Kosic, 2004; Berry, 2007; Martins y Reid, 2007).

Algunos de los primeros autores dando a conocer este término fueron Redfield, Linton, y Herskovits quienes en 1936 afirmaron que la aculturación es un fenómeno en el cual un grupo de individuos provenientes de diversas culturas establecen un contacto continuo y de primera mano con los miembros de la sociedad receptora. Como resultado de estas interacciones, ambos grupos experimentan cambios constantes y profundos en sus propios patrones culturales (Skuza, 2007). De la misma manera, Berry (1991) afirmó que la aculturación es un proceso de cambios culturales y psicológicos los cuales tienen un impacto directo en los individuos, las familias, y los diversos grupos culturales residiendo en un mismo territorio como consecuencia del contacto mutuo. El proceso en sí y los efectos del mismo son altamente variables generando diferencias muy marcadas a nivel individual, generacional y social. De esta manera, el proceso de aculturación es conceptualizado como un cambio significativo en la vida de los inmigrantes el cual conlleva una posible transformación de las actitudes, tradiciones, roles, interacciones sociales, conductas, y valores presentes en la cultura de origen. Para algunos inmigrantes la adaptación a una nueva cultura es un proceso entusiasta y fácil de llevar acabo; sin embargo, debido a las raíces que dejaron en sus países de origen, otros inmigrantes 
encuentran muchos obstáculos y desafíos en esta transición (Yeh et al., 2003).

\section{Adaptación sociocultural y psicológica}

Un elemento inmerso en el proceso de aculturación es la adaptación psicológica y sociocultural que los inmigrantes experimentan. A través de este proceso, los inmigrantes ajustan su vida a la nueva comunidad receptora con la finalidad de lograr una adaptación exitosa. En términos generales, Kim y Hurh (1993) propusieron tres elementos clave para entender el proceso general de adaptación. Para estos autores, la inmigración es una experiencia que conlleva el ajuste a un sinfín de elementos presentes en el contexto sociocultural. Al irse extendiendo el tiempo de residencia en la nueva sociedad, los inmigrantes necesitan desarrollar nuevas estrategias de adaptación basadas en los siguientes patrones de respuesta: (1) aceptación, (2) retención o (3) pérdida (Kim y Hurh, 1993).

Diversos estudios han identificado dos procesos de adaptación con características muy particulares. Por un lado está la adaptación psicológica la cual fomenta una evaluación positiva de las circunstancias, los logros personales, y el bienestar en general. Por otra parte, la adaptación sociocultural es definida como el aprendizaje social a través del cual los individuos son capaces de establecer relaciones constructivas con los miembros de la nueva cultura (Maydell-Stevens, Masgoret, y Ward, 2007). 
El proceso de adaptación psicológica abarca la inclusión de las estrategias de superación, los cambios en el estilo de vida, y la evaluación de los diferentes estresores, todo ello con la finalidad de favorecer un ajuste psicológico satisfactorio. La evidencia empírica ha revelado que distintos factores asociados con la adaptación psicológica tienen un impacto directo en la salud mental. De esta manera, los inmigrantes que han logrado una correcta adaptación psicológica se perciben a sí mismos no solamente de manera positiva siendo capaces de enfrentar apropiadamente los estresores de la vida cotidiana, sino también como valiosos y satisfechos (Neto, 1988). Por otra parte, la adaptación sociocultural está caracterizada por la adquisición de recursos de distinta índole como lo son la educación, los ingresos, la fluidez en el idioma, y la cantidad y calidad de las relaciones establecidas con los miembros de la nueva cultura. A su vez, la adaptación sociocultural está inversamente relacionada con la discriminación sufrida por los inmigrantes (Ward, 2004).

\section{Estrategias de aculturación}

Como ha sido mencionado, la adaptación a una nueva cultura es para la mayoría de los inmigrantes un gran desafío. En este proceso los inmigrantes no solamente necesitan aprender otras costumbres, leyes, idiomas, e interacciones sociales, sino que también necesitan adaptar sus ideas y comportamiento a la cultura dominante. A su vez, las estrategias de aculturación, las cuales son influenciadas por factores presentes en el contexto social así como variables rela- 
cionadas con la personalidad de los inmigrantes, son un indicador muy importante de la forma en que ellos interactúan con los miembros de la sociedad receptora (Berry, 2001). De esta manera, los inmigrantes son capaces de enfrentar los múltiples desafíos relacionados con el proceso de adaptación psicológica y sociocultural.

John W. Berry, quien es uno de los investigadores más reconocidos en temas relacionados con el fenómeno de la inmigración, propuso cuatro estrategias de aculturación. La primera es llamada "asimilación" y enfatiza las interacciones que los inmigrantes establecen con los individuos del país de residencia rechazando al mismo tiempo su cultura de origen. La segunda estrategia es la "separación" y ocurre cuando los individuos mantienen su cultura de origen y al mismo tiempo evitan interactuar con otros grupos étnicos, particularmente con el grupo mayoritario en el país de residencia. La tercera estrategia, "marginalización", está caracterizada por un interés casi nulo en la cultura de origen, (debido frecuentemente a la pérdida forzada de sus raíces culturales), así como la falta de interacciones con los miembros del país de residencia (debido frecuentemente a la exclusión o discriminación). Finalmente, la "integración" ocurre cuando los inmigrantes mantienen actitudes positivas hacia su cultura de origen y al mismo tiempo establecen relaciones significativas y respetuosas con los miembros del país de residencia. Esta última estrategia permite que los inmigrantes se adentren en la nueva cultura sin perder sus tradiciones y costumbres (Berry, 1997). 


\section{\begin{tabular}{l} 
arturo enrr que orozco vargas \\
\hline
\end{tabular}}

\section{La inmigración hispana en los Estados Unidos}

Aunque la inmigración es un fenómeno mundial, cada región geográfica tiene sus propias particularidades y la inmigración hispana a los Estados Unidos no es la excepción. Desde el siglo XVII, diferentes grupos étnicos han inmigrado a los Estados Unidos en muy distintas proporciones; sin embargo, durante las últimas cinco décadas el número de inmigrantes hispanos se ha incrementado de una manera sin precedentes alcanzando en el año 2010 un total de 40.2 millones de inmigrantes. Esta cifra incluye 29 millones de inmigrantes con un estatus legal (72\%) y 11.2 millones de inmigrantes indocumentados (28\%) (Passel y D'Vera Cohn, 2011). Al comienzo del siglo XXI, la población hispana viviendo en los Estados Unidos fue estimada en 35.3 millones de latinos (incluyendo inmigrantes documentados e indocumentados, residentes permanentes y ciudadanos americanos de origen hispano). De acuerdo con cifras del último censo en los Estados Unidos efectuado en el 2010, se espera que en el año 2050, aproximadamente el 25\% del total de la población en los Estados Unidos estará conformada por hispanos.

Aunque en el siglo Xx el número de inmigrantes latinos se incrementó año con año, a partir del año 2007, la tendencia ascendente que había prevalecido en la población inmigrante de origen latino viviendo en los Estados Unidos ha comenzado a disminuir a consecuencia de las tasas relativamente altas de desempleo en este país, las iniciativas de ley cada vez más restrictivas en contra de los inmigrantes indocumentados y la mejora en las condiciones econó- 
micas de algunos sectores productivos en ciertos países latinoamericanos. Esta tendencia ha sido confirmada por datos estadísticos mostrando que de los 11.5 millones de inmigrantes indocumentados quienes estaban residiendo en el año 2011, el 55\% de ellos entraron a este país entre los años 1995 y 2004 contrastando con el 14\% de inmigrantes indocumentados que entraron entre el 2005 y 2011 (Hoefer, Rytina, y Baker, 2011). Considerando exclusivamente a las mujeres, durante los años 2005 y 2006 alrededor de 105 mil mujeres provenientes de países latinoamericanos inmigraron a los Estados Unidos (Solís-Pérez y Alonso-Meneses, 2009).

Dado el predominio de la inmigración hispana y el porcentaje de familias latinas viviendo en los Estados Unidos, el objetivo de este estudio cualitativo es investigar desde una perspectiva fenomenológica el significado que tienen las experiencias de aculturación de un grupo de ocho mujeres hispanas quienes inmigraron a los Estados Unidos. Para lograr dicho cometido, el método fenomenológico fue elegido para analizar el significado de las experiencias de estas mujeres inmigrantes. A través de su discurso, ellas describieron no solamente la realidad que estaban viviendo al momento de realizar las entrevistas, sino también su participación en actividades cotidianas como lo son la crianza de sus hijos, su trabajo fuera de casa, sus estudios, las compras que hacen, los pagos de distintos servicios y todas las actividades que ellas desempeñan dentro y fuera de su hogar. De esta manera fue posible entender la manera en la cual las mujeres inmigrantes han internalizado sus experiencias de aculturación como madres, esposas, e hijas. 


\section{Metodología}

Los principios teóricos de la fenomenología fueron usados en el presente estudio con la finalidad de lograr una mayor comprensión de las experiencias de vida de las ocho mujeres quienes participaron en esta investigación. Se trata de un método filosófico cuyo propósito es descubrir y describir la esencia de las experiencias humanas. Este aspecto es central para la fenomenología y puede remitir al conocimiento de los valores, actividades, sentimientos, objetos físicos y los estados de ánimo (Daly, 2007). Tiene sus raíces en la filosofía de Husserl quien a su vez basó sus principios teóricos en Franz Von Brentano y señaló que la fenomenología estudia las experiencias de la conciencia. Años más tarde, Jaspers introdujo en la psicopatología y la psiquiatría el método fenomenológico, que describe las experiencias subjetivas de los individuos (Ruiz y Cano, 2002). El objetivo de Husserl era desarrollar una filosofía que fuera metodológicamente independiente y que pudiese comenzar sin prejuicios. Durante el siglo Xx, la fenomenología influenció a través de Scheler en la antropología filosófica, a través de Heidegger en la hermenéutica, y a través de Schütz en la filosofía social. Los representantes más importantes de este método son Scheler, Heidegger, Jaspers, Merleau-Ponty y Sartre (Hoffe, 2003).

El propósito de la investigación fenomenológica es escudriñar en la realidad de los individuos con la finalidad de comprender los significados personales que construyen desde sus experiencias de vida. De esta manera es posible conocer lo que cada experiencia 
en particular significa para ellos (Johnson y Christensen, 2008). A su vez, Skuza (2007) afirmó que el objetivo de este método es revelar el significado fundamental de un fenómeno dentro del contexto en el cual los individuos lo experimentan, así como distinguir los rasgos de ese fenómeno. En términos generales, estos estudios analizan el significado que los individuos atribuyen a sus experiencias de vida relacionadas a un fenómeno o a un concepto en particular. Por medio de los estudios fenomenológicos es posible conocer aquello que los participantes comparten en común mientras ellos experimentan un fenómeno (Creswell, 2007).

Basado en dicho método, el objetivo de esta investigación fue identificar y analizar los motivos que llevaron a estas inmigrantes a dejar sus países de origen y las principales experiencias relacionadas con su propio proceso de aculturación.

\section{Participantes}

Ocho mujeres hispanas quienes inmigraron a los Estados Unidos fueron invitadas a participar en este estudio. Para ser incluidas en él, las participantes tuvieron que: (1) ser mujeres hispanas con una edad entre los 25 y los 60 años, (2) mujeres que nacieron en algún país latinoamericano, y (3) mujeres que inmigraron a los Estados Unidos por lo menos hace 5 años. La muestra estuvo compuesta de ocho, cuya residencia estaba localizada en el Metroplex de Dallas, Texas. Todas las participantes estaban casadas con excepción de una que se había divorciado de su esposo. Sus edades oscilaron 
entre los 27 y los 53 años. Con respecto al número de hijos, tres de ellas tenían dos hijos, dos de ellas tenían cuatro hijos, una tenía tres hijos, una tenía un hijo, y una de las entrevistadas no tenía hijos. Al momento de la entrevista, la participante con menos años viviendo en los Estados Unidos tenía 7 y la participante con más años tenía 42. Con respecto al nivel educativo, solamente una había concluido sus estudios de licenciatura, 3 de ellas lograron terminar la preparatoria, 2 de ellas tenían estudios de secundaria y 2 de ellas no alcanzaron a terminar la primaria.

\section{Recolección de datos}

Los procedimientos de recolección de información que se presentan a continuación se encaminan a la obtención fenomenológica del proceso de aculturación que han vivido las ocho mujeres que tomaron parte en este estudio. Las participantes fueron seleccionadas a través de un muestreo de conveniencia y de bola de nieve. Después de haber contactado a la primera de ellas, ésta me proporcionó la información necesaria para contactar a la siguiente y así sucesivamente hasta alcanzar el total de las ocho participantes. Todas fueron contactadas por teléfono y luego de informales acerca del propósito general del estudio, se les hizo la invitación directamente a participar en él. Después de haber aceptado, se fijó una cita para llevar a cabo la entrevista. Todas las entrevistas se realizaron en sus casas, esto ayudó a que se sintieran cómodas y no tuvieran la necesidad de viajar a otro sitio para ser entrevistadas. 
El procedimiento empleado en cada una de las entrevistas fue muy semejante; antes de comenzar, se les explicó en detalle el objetivo del estudio, los procedimientos generales y sus derechos como participantes. Finalmente se les pidió su autorización para audiograbar la entrevista. De esta manera, ocho entrevistas fenomenológicas fueron llevadas a cabo con una duración de aproximadamente una hora y media; la que menos tiempo duró fue de 70 minutos y la de más tiempo tuvo una duración de una hora 55 minutos.

\section{Instrumento}

Para llevar a cabo las entrevistas se diseñó un protocolo semiestructurado; el cual contenía 19 preguntas basadas en el significado de las experiencias relacionadas con los motivos que tuvieron para emigrar, y principalmente con el proceso de aculturación que cada participante ha vivido en los Estados Unidos. Aunado a estas preguntas generales, un promedio de 30 preguntas específicas se le hicieron a cada participante con la finalidad de describir a detalle ciertas experiencias particulares que han distinguido su estadía en este país. Basado en lo anterior, se pensó en emplear el formato semiestructurado porque ofrece la posibilidad de llevar a cabo una investigación exploratoria y realizar preguntas adicionales cuando es necesario para clarificar algunos temas (Yeh et al., 2003). El protocolo fue diseñado después de haber completado una revisión exhaustiva de la literatura científica y fue dividido en 3 secciones. La primera tenía por objetivo conocer los distintos motivos que las 
llevaron a emigrar así como las condiciones familiares y laborales que tenían en su país de origen; la segunda sección cuestionó su proceso de adaptación psicológica y sociocultural a los Estados Unidos, y la última sección incluyó preguntas con respecto a las estrategias, recursos y barreras que habían enfrentado en su nueva estadía.

\section{Análisis de datos}

Los datos provenientes de la narrativa de las participantes fueron analizados usando el método "Investigación Cualitativa Consensual" (CQR por sus siglas en inglés) que es un procedimiento metodológico diseñado por Hill, Thompson, y Williams. Este método está basado en el estudio intensivo de pocos casos, usando un proceso inductivo-analítico (Hill, Thompson, y Williams, 1997). De acuerdo con estos autores, una vez que las entrevistas son transcritas, los datos recopilados en ellas son analizados a través de tres fases. La primera etapa pide que los investigadores dividan los datos en dominios. Estos dominios representan las áreas generales o tópicos, los cuales agrupan todos los datos puros incluidos en las entrevistas. En el siguiente paso, los investigadores revisan las transcripciones con la finalidad de modificar la primera lista y categorizar el contenido en dominios.

La segunda etapa involucra la abstracción de las ideas núcleo. En esta fase los investigadores desarrollan una serie de ideas núcleo con la finalidad de sintetizar los datos incluidos en cada domi- 
nio considerando cada caso de manera particular. De acuerdo con el método CQR, este paso implica la discusión de los comentarios más significativos de los participantes hasta que los investigadores llegan a un consenso proponiendo una versión final de los dominios e ideas núcleo. La última etapa involucra un análisis integral cuyo objetivo es desarrollar las categorías que emanan de las ideas núcleo presentes en cada narrativa. Hill y sus colaboradores señalaron que el objetivo de este paso es llevar a cabo un análisis transversal a través del cual las ideas núcleo son incorporadas en categorías. Esta fase concluye cuando los investigadores llegan a un consenso el cual asegura que los datos puros han sido codificados apropiadamente (Hill et al., 1997).

\section{Presentación de los resultados}

La gran mayoría de los estudios usando el método CQR incluyen una tabla que refleja la frecuencia de las categorías presentes en la muestra. De acuerdo con Hill y colaboradores (1997), aquellas categorías que se aplican a todas las participantes en la muestra son llamadas categorías "generales". Las categorías presentes en la mitad o más de las narrativas son consideradas "típicas". Finalmente, las categorías que son identificadas en menos de la mitad de la muestra se llaman "variables". La Tabla 1 da a conocer la clasificación de las categorías presentes en este estudio. 


\section{Resultados}

Basados en el método CQR, se llevó a cabo un análisis fenomenológico con la finalidad de conocer en profundidad las experiencias de vida de las ocho inmigrantes hispanas que participaron en este estudio. A través de este análisis se identificaron los principales factores asociados con el significado del proceso de aculturación y las condiciones previas a éste. Cinco dominios fueron identificados y se muestran en la Tabla 1; estos son: (1) razones para emigrar, (2) desafíos y obstáculos, (3) beneficios y logros, (4) la barrera del idioma inglés, y (5) recursos.

A su vez, cada dominio incluyó varias categorías describiendo el fenómeno de la aculturación, los cambios positivos y negativos, las personas e instituciones que ayudaron en este largo proceso, los miedos y preocupaciones, el contacto familiar, las nuevas costumbres y normas, las oportunidades de trabajo y educación, el apoyo familiar y de pareja, la relación con otros latinos, la falta de fluidez en el idioma, y el estatus migratorio, así como el impacto de cada uno de estos factores en la vida de las inmigrantes hispanas.

\section{1) Razones para emigrar}

En las ocho entrevistas llevadas a cabo, todas las participantes coincidieron al mencionar que la principal razón por la cual emigraron fue la búsqueda de mejores condiciones económicas en los Estados Unidos. Aunque cuando estuvieron viviendo en México, dos de 
$214 \longrightarrow$ LAVENTANA, NÚM. $41 / 2015$

Tabla 1. Dominios y Categorías

\begin{tabular}{|c|c|c|}
\hline & Dominio/Categoría & Frecuencia \\
\hline \multirow[t]{4}{*}{ 1. Razones para emigrar } & Mejores oportunidades económicas & General \\
\hline & Disponibilidad de trabajo & General \\
\hline & Educación para los hijos & Típica \\
\hline & Familiares ya viviendo en los EEUU & Típica \\
\hline \multirow[t]{10}{*}{ 2. Desafíos y obstáculos } & Sentimientos de nostalgia & General \\
\hline & Adaptación inicial & General \\
\hline & Miedo a la deportación & Típica \\
\hline & Incapacidad de dejar el país & Típica \\
\hline & Nuevas costumbres & General \\
\hline & Situación de hijos no nacidos en EEUU & Típica \\
\hline & Ajuste profesional & Típica \\
\hline & Problemas con adolescentes & Variable \\
\hline & Porvenir después de la jubilación & Variable \\
\hline & Preocupaciones con respecto a los hijos & Típica \\
\hline \multirow[t]{7}{*}{ 3. Beneficios y logros } & Contacto familiar & General \\
\hline & Metas personales & General \\
\hline & Oportunidad de trabajar fuera del hogar & Típica \\
\hline & Cambio de actitudes & Variable \\
\hline & Hijos bilingües & General \\
\hline & Proceso de adaptación & General \\
\hline & Recompensas por los sacrificios hechos & Variable \\
\hline \multirow[t]{4}{*}{ 4. La barrera del idioma inglés } & Falta de fluidez en el idioma & General \\
\hline & Frustración & Típica \\
\hline & Estancamiento profesional & Variable \\
\hline & Preocupaciones con respecto a la comunicación & General \\
\hline \multirow[t]{7}{*}{ 5. Recursos } & Apoyo familiar & General \\
\hline & Apoyo de la pareja & General \\
\hline & Comunicación con familiares en el país de origen & Típica \\
\hline & Contacto con latinos & General \\
\hline & Voluntariado en la escuela de los hijos & Variable \\
\hline & Amigos y vecinos & Típica \\
\hline & Clases de inglés & General \\
\hline
\end{tabular}


ellas tuvieron un nivel socioeconómico medio, la oportunidad de incrementar su situación económica las motivó a dejar su país de origen. Asimismo, para cinco de ellas quienes tenían un nivel socioeconómico extremadamente bajo, el haber inmigrado a los Estados Unidos ha representado un cambio radical para sus vidas y las de sus hijos. De esta manera, no solamente todas las participantes han podido satisfacer sus necesidades básicas, sino que también ellas han obtenido otros recursos económicos que jamás hubieran conseguido en sus comunidades de origen:

Yo me vine para trabajar porque en México la economía no está muy bien. Las ilusiones de mi esposo y mías eran venirnos, trabajar un rato, gastar lo menos y ahorrar lo más que se pudiera para construir una casita en México (Inmigrante mexicana, 32 años).

Otra de las participantes nos compartió:

Yo inmigré a los Estados Unidos porque aquí vive una hermana mía, y ella se sentía muy sola. Y ella fue el gancho para estarme jalando y decirme vente, acá hay otras oportunidades, mejor vida. Y eso fue lo principal que nos hizo estar en este país (Inmigrante mexicana, 45 años).

La segunda razón que mencionaron las participantes fue la mayor oferta de trabajo. En todos los casos de las mujeres casadas, sus 
esposos pudieron conseguir trabajo de manera inmediata lo cual fue determinante para satisfacer sus necesidades básicas durante los primeros meses de su residencia en los Estados Unidos. Después de algunos meses, seis de las participantes consiguieron un trabajo gracias al cual la economía familiar ha podido ser más estable. Aunque el tener un trabajo en la fuerza laboral conlleva un gran esfuerzo por el tiempo y la distancia que necesitan recorrer, ellas mencionaron que se sienten muy satisfechas por ayudar a su esposo con los gastos familiares:

El trabajar limpiando casas ha sido muy importante para mí, la posibilidad de ganar algo de dinero haciendo algo que he hecho desde antes de casarme. Gracias al trabajo les puedo mandar dinero a mis hermanas y con eso me siento muy bien. Y eso hace que le eche más ganas de estar aquí. Uno desde que se viene al norte necesita estar trabajando, aunque el esposo trabaje, uno necesita trabajar para sentirse útil, para ganar algo de dinero, para que se pase el tiempo, por todo. También es importante el que sepa manejar, entonces de esa manera yo ya me voy a mis trabajos (Inmigrante mexicana, 28 años).

Finalmente, todas las participantes casadas coincidieron al decir que una de las razones más importantes para emigrar está relacionada con el mejor nivel educativo que sus hijos pueden alcanzar en este país. De manera particular, para aquellas que vivían en zonas rurales, sus hijos tienen ahora la posibilidad de conseguir un futuro educativo inimaginable. 


\section{culos}

\section{2) Desafíos y obstáculos}

Todas las participantes coincidieron al mencionar que la experiencia de inmigración ha tenido aspectos tanto positivos como negativos. Durante el largo y complicado proceso de aculturación, todas las participantes se vieron expuestas a grandes desafíos. Las entrevistadas afirmaron que de manera particular los primeros meses fueron realmente difíciles para ellas y para aquellos quienes las acompañaron en su camino hacia los Estados Unidos. Al llegar a este país, cuatro de las participantes llegaron a vivir a la casa de sus parientes. Aunque durante las primeras semanas el estar cerca de su familia les ayudó mucho, al cabo de los meses esta situación se tornó muy incómoda:

Como llegamos a vivir con su hermano (del esposo), yo tenía que compartir la estufa y pensaba que en México yo tenía mi propia estufa. Lo más difícil fue el haber estado con ellos, pues aunque nos ayudaron mucho, fue muy duro el compartir todo. Además ellos tenía otras costumbres y uno se sentía incómodo. Además ella (la concuña) amanecía algunos días enojada, y yo quería salirme para no verlos. Hasta que nos mudamos a la primera casa que pudimos rentar, entonces si fue un cambio radical. Yo no pensé llegar y compartir la casa con otra familia, entonces eso fue bien difícil" (Inmigrante colombiana, 38 años). 
Con respecto a otros desafíos y obstáculos que conlleva el proceso de aculturación, las participantes acentuaron los sentimientos de nostalgia por sus familiares que dejaron atrás, el miedo a ser deportadas, y el saber que no podrán salir de los Estados Unidos. Esta situación ha traído una gran tristeza para ellas pues están conscientes que resulta imposible regresar a sus países de origen con la finalidad de visitar a sus familias y amistades:

La primera preocupación es que estás pensando en que te deporten, otra es que no puedes ir a visitar a tu familia cuando quieres, porque es fácil irte pero no está fácil para regresarte (Inmigrante mexicana, 53 años).

Otro de los grandes desafíos tiene que ver con la situación de los hijos que no nacieron en los Estados Unidos lo cual siempre es motivo de mucha preocupación para ellas. En particular, tres de las participantes tienen uno o dos hijos que nacieron en México; por consecuencia, aunque son niños, esta condición no los exenta de ser en alguno momento deportados:

Una de las cosas que más me afecta es que mi hija pequeña como nació en los EEUU tiene papeles, pero en cambio la niña grande no puede salir. Entonces se me hace muy incómodo que mi niña la más grande no tenga papeles, entonces cuando se trata de ir a México ella no puede ir y eso se me hace muy discriminativo e injusto hacer eso con mis propias hijas. Y 
que la niña grande crezca con eso en su mente, pensando que su hermana puede ir y ella no porque es mojada. Y ella todo el tiempo me pregunta por qué no puede ir y mi hermana sí puede ir. Se me hace algo muy triste que solamente por no haber nacido aquí tengan que vivir una situación diferente (Inmigrante mexicana, 27 años).

Además de ello, las participantes expresaron serias preocupaciones relacionadas con el bienestar de sus hijos, destacando su salud, su adaptación a la cultura norteamericana, la influencia que ellos reciben de sus amigos y compañeros de clase, y sobre todo su situación académica cuando alcancen el nivel superior. De manera particular, dos de las participantes han vivido situaciones muy conflictivas y estresantes con sus hijos ya adolescentes. Aunque estas participantes no tuvieron problemas con sus hijos durante su niñez, al llegar a la adolescencia la frecuencia de los conflictos entre padres e hijos se incrementó significativamente:

Con mi hijo hasta los 18 años yo lo podía dominar de todo, de llegar a tal hora o de ir a algún lado, pero cuando cumplió los 18, el dio una vuelta que hasta lo desconocí, un giro bien drástico como diciendo ya tengo 18 años puedo hacer lo que a mí me dé la gana (Inmigrante salvadoreña, 49 años).

Finalmente, otro grupo de desafíos está compuesto por la adaptación a las costumbres norteamericanas, la falta de un número de 
seguro social para poder trabajar, y su porvenir una vez que dejen de trabajar. De manera particular, dos de ellas tienen estudios de nivel superior; sin embargo, debido a la falta de fluidez en el idioma inglés y su estatus migratorio, no han podido conseguir un trabajo relacionado con los estudios que cursaron.

\section{3) Beneficios y logros}

A pesar de los obstáculos y desafíos ligados a su nueva estadía en los Estados Unidos, todas las participantes señalaron que el proceso de aculturación ha traído grandes beneficios. Uno de los más importantes para ellas es que sus hijos son bilingües. A través de la conversación diaria que sostienen en su casa, los niños aprendieron el español y después de pasar algunos meses en la escuela, los niños comienzan a hablar en inglés de manera natural. Es así como los niños pueden expresar sus ideas y sentimientos en español e inglés con lo cual incrementarán en el futuro sus oportunidades de alcanzar mayor nivel educativo y profesional que el de sus padres.

Otro aspecto positivo está relacionado con el hecho de sentirse más cómodas y haberse acostumbrado a la forma de vida en los Estados Unidos. Es verdad que los primeros meses fueron muy difíciles y llenos de miedos; sin embargo, gracias al apoyo de su familia y amigos ellas comenzaron a superar esos temores:

La familia del hermano de mi esposo fue muy importante al inicio para que yo me empezara a sentir más segura y no me 
diera tanto miedo cuando salíamos a comprar cosas o ver a las maestras a la escuela de mis hijos y ahora con familiares de mi esposo quienes también nos echan la mano. Principalmente con vecinas y amigas quienes he ido haciendo aquí en el parqueadero donde vivimos, son muy buenas y les tengo confianza para pedirles algún favor cuando lo necesito mucho. Aquí, todo el tiempo ocupa uno de la gente y en cualquier cosa que se ofrezca siempre hay alguien que nos ayuda (Inmigrante mexicana, 32 años).

Otros dos beneficios están relacionados con la posibilidad de trabajar en la fuerza laboral y algunos cambios personales de su esposo. De manera particular, a tres de las participantes, cuyos esposos tenían ideas machistas muy fuertes, no se les había permitido trabajar fuera del hogar; sin embargo, desde que están viviendo en los Estados Unidos, ellos han cambiado su manera de pensar y ahora con su apoyo, tienen un trabajo con el cual contribuyen de manera significativa a la economía familiar:

El trabajo ha sido de gran beneficio para mí, porque yo me pongo a pensar en qué triste es para las mujeres allá (en México) desde que se levantan puro quehacer y quehacer todos los días, y después llega el fin de semana y no reciben ningún dinero a cambio. Por el contrario, los fines de semana los hombres se salen a tomar con los amigos y en cambio las mujeres encerradas en su casa (Inmigrante mexicana, 40 años). 
Los beneficios obtenidos como fruto de su residencia en los Estados Unidos también tocan directamente el área familiar. Por ejemplo, tres de las participantes mencionaron que después de haber comenzado a residir en los Estados Unidos, los miembros de su familia pueden pasar más tiempo juntos. Por consecuencia, sus lazos familiares se han estrechado considerablemente:

Pienso que el haber inmigrado ha sido exitoso como al 80\%. Por una parte mi esposo y yo hemos conseguido el convivir más porque en México respecto a nuestros trabajos durábamos mucho tiempo sin vernos. Mientras que yo estaba trabajando todo el día en la oficina y salía como a las 6 de la tarde, llegaba a mi casa y cuando a mi esposo le tocaba trabajar el turno de la tarde lo veía hasta las 11 de la noche. Si le tocaba trabajar en la noche yo solamente lo podía ver un par de horas hasta que él se iba a trabajar. Solamente los domingos los teníamos para nosotros y visitar a la familia. Así que uno de nuestros logros aquí en los Estados Unidos ha sido el convivir más y salir de nuestros compromisos económicos (Inmigrante mexicana, 53 años).

Finalmente, después de haber hecho un sinfín de sacrificios, no solamente reciben las primeras recompensas, sino que también tienen la posibilidad de soñar con metas y proyectos a mediano y largo plazo tanto para ellas mismas como para sus familias: 
Hoy en día, yo me siento bien, me siento contenta pues ya tenemos esta casita y a lo mejor más adelante pensamos en algo mas grande, pero al pasito. Como va pasando el tiempo uno se va acomodando y le va dando gusto que si va uno avanzando y ya no le va dando el miedo que a uno antes sí le daba (Inmigrante mexicana 32 años).

\section{4) La barrera del idioma inglés}

El idioma inglés fue considerado un dominio porque todas las participantes señalaron que la falta de fluidez en el idioma es un factor extremadamente importante en el proceso de aculturación. Debido a que siete de las participantes no tuvieron oportunidad de estudiar inglés antes de emigrar hacia los Estados Unidos, ellas han experimentado innumerables obstáculos así como un gran sentimiento de frustración cuando quieren expresar sus ideas, o bien al no poder entender lo que la gente les dice:

El idioma siempre ha sido un problema; por ejemplo cuando nació mi segunda hija, en el hospital no había nadie que hablara español y era difícil decirles lo que me pasaba o que me fueran a decir algo y que nos les entendiera, para mí fue desesperante. También aquí en la escuela donde están mis niñas nadie habla español, así que se me dificulta mucho pues entiendo unas cosas y otras no. Y quisiera estar involucrada en algunas actividades y pues no puedo porque no sé el idioma. 
O con mi hija pequeña que se le dificultan algunas cosas en sus tareas pues hay veces que no puedo pues no las sé en inglés (Inmigrante colombiana, 38 años).

Todas las participantes coincidieron al afirmar que si el idioma inglés hubiese sido su lengua materna, su situación en este país sería completamente diferente. La barrera del idioma tiene muchos efectos negativos sobre la vida de las inmigrantes; sin embargo, uno muy especial está relacionado con su situación laboral. Tres de las participantes, quienes tienen estudios de nivel medio superior, comentaron que debido a la falta de fluidez cuando ellas hablan en inglés, no han podido conseguir un mejor trabajo. Aunque estas participantes llevan ya trabajando varios años en la misma compañía, desafortunadamente no han podido crecer dentro de ella pues para lograrlo necesitarían tener un nivel de inglés más elevado:

El idioma siempre ha sido el principal problema, de ahí han venido otros problemas, por ejemplo, el hecho de no poder conseguir un mejor trabajo, pues el idioma es fundamental. Para mí ha sido difícil pues he tenido que comenzar desde abajo en el restaurant (Inmigrante mexicana, 28 años).

Otra participante comentó:

Como en México estaba trabajando y cuando llegué a los EEUU no pude trabajar por el idioma, al principio me sentía frustra- 
da pues me preguntaba de qué había servido estudiar y trabajar en México. Mi idea es trabajar en los EEUU, quiero estudiar y prepararme pues quiero trabajar en oficina. Al principio extrañaba mi trabajo muchísimo, yo deseaba estar en mi trabajo y pensaba que mejor me hubiera quedado en México porque yo amaba mi trabajo. Además como convivía con mucha gente, al principio me afectó pues al no conseguir un trabajo por consecuencia estaba aquí encerrada en la casa (Inmigrante mexicana, 45 años).

La misma situación ocurre cuando tienen que entrevistarse con alguna maestra de sus hijos que solamente habla inglés o cuando tienen que ir a pagar o solicitar un servicio en una agencia gubernamental. La mayoría de las participantes mencionó que ellas pueden expresar algunas ideas en inglés y entender frases muy básicas; sin embargo, les es muy difícil entender detalles muy específicos en una conversación:

El principal obstáculo ha sido la barrera del idioma, por ejemplo cuando voy al doctor y no hay quien te ayude en español ha sido un problema pues no entiendo o yo entiendo una cosa y me están diciendo otra cosa. Para mí, mi gran problema es el idioma. Y muchos otros problemas se generan a partir del idioma (Inmigrante salvadoreña, 49 años). 


\section{5) Recursos}

Todas las participantes señalaron que desde el momento que abandonaron su país de origen, ciertos recursos han sido determinantes para enfrentar el sinfín de barreras y desafíos encontrados en el camino. Entre los más importantes para ellas está el apoyo familiar y el de su pareja. Para aquellas participantes quienes después de haber llegado a los Estados Unidos recibieron un apoyo constante de parte de sus parientes, éste ha sido el mejor recurso para lograr una adaptación satisfactoria sobre todo durante los primeros meses que resultan ser los más difíciles:

Los primeros 6 meses fueron de adaptación, en ese tiempo mi hermana me apoyó muchísimo, nos consiguieron un departamento para rentar y yo creo que ella fue quien me impulsó a no sentirme tan mal. Al principio extrañaba todos los días a mis papás pues ya son grandes y me preocupaba sobre todo por la salud de mi mamá; sin embargo, mi esposo platicaba conmigo y me decía que mi otra hermana iba a estar al pendiente de ellos y que con el paso de los meses ya me iba a sentir mejor. La verdad es que de no haber sido por mi hermana y su cuñado no sé cómo le habría hecho para transportarme, para saber dónde pagar los servicios, para inscribir a mi hija en la escuela. Incluso mis sobrinos también han sido muy importantes pues al llegar aquí mi hija tuvo la oportunidad de con- 
vivir con ellos y no sentirse mal por extrañar a sus amiguitos que tenía en su escuela (Inmigrante mexicana, 40 años).

A su vez, todas las participantes, quienes se encuentran casadas, expresaron que la decisión de emigrar a los Estados Unidos fue hecha bajo consentimiento mutuo. Aunque en la mitad de los casos, uno de ellos fue el que propuso explícitamente la idea de irse a los Estados Unidos, después de pensarlo y consultarlo con sus familiares y amistades, todas las participantes casadas decidieron acompañar voluntariamente a su pareja en esta aventura. Un caso ilustrativo es el siguiente:

La decisión de venirnos para acá fue de mutuo acuerdo. Jorge (esposo) tomó la primera decisión de isabes qué?, vámonos, nos están ofreciendo mejores oportunidades; mi cuñado le ofrecía a mi esposo un trabajo. Yo le dudé un poquito porque sabía que era un poco difícil estar aquí, pero entre los dos tomamos la decisión y estuvimos de acuerdo (Inmigrante colombiana, 38 años).

Por otra parte, con el propósito de mantener una constante y cercana relación con sus familiares viviendo en el país de origen, todas las participantes mencionaron que ellas llaman frecuentemente a sus papás, hermanos, y demás familiares con el propósito de mitigar los sentimientos de nostalgia y tristeza causados por la separación: 
También el hablar por teléfono, ya cuando hablo con alguien de mi familia siento que estoy con ellos. Y hablando seguido, siento que estoy cerca de ellos, casi siento que los estoy mirando. Desde que llegué todo el tiempo estoy en comunicación con mi familia y en algunos casos les mando una cosita (Inmigrante mexicana, 32 años).

En el discurso de las participantes, otro recurso muy citado fue el contacto con latinos viviendo cerca de ellas, así como el apoyo recibido por parte de amigos y vecinos. Todas las participantes de una u otra manera mencionaron que en sus actividades diarias como lo son la crianza de los hijos, llevarlos a la escuela, las labores domésticas, y las compras, ellas siempre han recibido una ayuda constante de la comunidad hispana. De la misma manera, la mayoría comentó que en las distintas ocasiones en las cuales la escuela donde asisten sus hijos ha pedido voluntarias para ayudar con las actividades escolares, ellas tratan de participar en todos los eventos que convocan. Con ello, estas entrevistadas tienen no solamente la oportunidad de practicar su inglés, sino que también, en estas actividades pueden conocer a otras madres con quienes, a través del contacto diario, terminan siendo muy buenas amigas:

Otro cambio ha sido el relacionarme más en la escuela de mis hijos pues antes no me gustaba, tenía miedo por la barrera del idioma y ahora ya me mezclo más, me gusta ser voluntaria. Ya estoy perdiendo el miedo del idioma (Inmigrante mexicana, 27 años). 
Finalmente, las clases de inglés han sido un factor esencial en su proceso de aculturación. Seis de las participantes han asistido a clases de inglés con lo cual han incrementado de manera significativa sus habilidades de comunicación. Estas clases les han ayudado sobre todo a entender de mejor manera lo que otras personas les dicen y a perder el miedo cuando tienen que hablar con algún norteamericano:

Al llegar a los Estados Unidos uno no sabe nada de inglés, allá en el rancho nunca nos enseñaron nada, entonces cuando uno llega aquí de grande no entiende nada de lo que le dicen. Al principio por más que ponía atención se me hacía imposible entender, ya después poco a poco y sobre todo con las clases que he tomado de inglés ya les comienzo a entender más fácil. Sobre todo con mis patronas cuando me dicen lo que quieren que les limpie o los arreglos que quieren que haga al menos ya no me siento tan perdida. Así que aunque sea ya de noche y tengo que dejar cenados a mis hijos y listos para que su papá los mande a dormir, esas clases han sido de mucha ayuda para que yo me defienda mejor en eso del inglés (Inmigrante mexicana, 40 años).

\section{Discusión}

Como innumerables autores han afirmado, el fenómeno de la aculturación es tan complejo que nos da la oportunidad de investigarlo desde diversas perspectivas. En el presente estudio cualitativo, ocho 
inmigrantes hispanas ofrecieron una descripción exhaustiva de su propia experiencia de aculturación y los motivos que tuvieron para emigrar a los Estados Unidos. Los detalles de estas narrativas son presentados en la Tabla 1. Después de haber analizado el discurso de las participantes, es posible llegar a varias conclusiones. La primera de ellas tiene que ver con aquellas circunstancias previas al momento de emigrar. De acuerdo con las participantes, la razón principal que tuvo este grupo de mujeres para haber dejado su país de origen fue el deseo de alcanzar mejores condiciones económicas. Estudios recientes han señalado que si bien es cierto que algunos de los inmigrantes que se establecen en los Estados Unidos lo hacen por un periodo de tiempo relativamente corto con la finalidad de mejorar su situación económica y regresar entonces a sus países de origen; la inmensa mayoría de estos trabajadores busca una residencia permanente en este país. A este respecto, Ogbu (1990) afirmó que a pesar de los riesgos que conlleva, los inmigrantes dejan sus comunidades de origen con la convicción de encontrar mejores oportunidades de vida para ellos y sus familias.

A su vez, el hecho de que algunos familiares de la mayoría de las participantes estuvieran ya viviendo en los Estados Unidos fue considerado otro de los principales motivos para viajar. De acuerdo con Boneva y Frieze (2001) la decisión de emigrar es más frecuente cuando los familiares y amigos que ya se encuentran viviendo en el país receptor los instan a emigrar. Este fue el caso de siete de las participantes quienes fueron invitadas directamente por sus familiares para establecerse cerca de ellos. 
Una segunda conclusión tiene que ver con los desafíos que los inmigrantes encuentran una vez ya establecidos en los Estados Unidos. Todas las participantes señalaron que su experiencia de aculturación fue sumamente difícil durante los primeros meses. A este respecto, Adelman (1988) señaló que la primera vez que los inmigrantes se ven expuestos en la nueva cultura, la adaptación puede ser difícil porque se topan con exigencias y normas totalmente nuevas para ellos. De manera particular, las entrevistadas mencionaron que la adaptación a las nuevas costumbres norteamericanas ha sido el desafío más grande. Esta tendencia es consistente con los resultados encontrados por Martins y Reid (2007) los cuales mostraron que aquellas mujeres que emigraron a Canadá provenientes del sur de Asia tuvieron muchos problemas para lograr una adaptación satisfactoria por las diferencias muy marcadas entre sus propios valores familiares y culturales y aquellos arraigados en la cultura canadiense.

Por otra parte, la tercera conclusión está relacionada con los beneficios obtenidos. El discurso de las participantes estuvo lleno de ejemplos que dan cuenta de los aspectos positivos que conlleva el proceso de aculturación. Entre ellos, la mayoría de las participantes exaltaron los grandes beneficios que han obtenido desde que comenzaron a trabajar fuera de su hogar. Este mismo patrón fue encontrado por Shin (1999) quien mostró que para las inmigrantes coreanas la oportunidad de trabajar, en la fuerza laboral, fue un factor decisivo no solamente para proveer ayuda financiera al hogar, sino también para ser más conscientes de sus derechos 
como mujer, lograr una mayor independencia, y confiar más en ellas mismas.

Otra de las principales conclusiones derivadas del discurso de las participantes enfatizó lo que ellas describieron como la barrera del idioma inglés. Todas las participantes afirmaron que la falta de fluidez en el idioma inglés fue el principal obstáculo en su proceso de aculturación; por ende, esta situación ha traído consecuencias muy negativas en su adaptación a la cultura norteamericana. A este respecto, George y Ramkissoon (1998) mencionaron que las mujeres que son incapaces de escribir y hablar en inglés con soltura les es sumamente difícil afrontar las tareas y obligaciones que demanda el proceso de adaptación a la nueva cultura. Los resultados del presente estudio muestran que incluso aquellas que han podido tomar clases de inglés y mejorar su fluidez, no se ven exentas de las dificultades más comunes.

Finalmente, las entrevistadas describieron la importancia del apoyo que han recibido de sus familiares, amigos, y vecinos, así como el contacto con otros latinos volviéndose fundamental en su proceso de aculturación. De la misma manera, en otro estudio cuyo objetivo fue identificar problemas comunes relacionados con la adaptación psicológica y sociocultural, Maydell-Stevens y colaboradores (2007) encontraron que el apoyo social fue un factor determinante para que un grupo de inmigrantes rusos pudiera adaptarse satisfactoriamente en Nueva Zelanda. En este estudio, los miembros de la familia extensa proveyeron el apoyo emocional y los recursos materiales necesarios para estos inmigrantes. 
Semejante a otras investigaciones de corte cualitativo, en el presente estudio es posible identificar algunas limitaciones. La primera de ellas tiene que ver con el lugar de residencia, pues todas las participantes se encuentran viviendo en el norte de Texas. De esta manera, los resultados solamente muestran cómo el fenómeno de la aculturación está presente en esta área geográfica en particular. Por otra parte, como fue señalado en la descripción de las participantes, todas las mujeres excepto una están casadas y tienen hijos. Por consiguiente, es necesario examinar cómo el proceso de aculturación puede ser diferente para mujeres solteras y sin hijos y de esa manera comparar los resultados con los obtenidos en este estudio. Finalmente, como sucede en la mayoría de los estudios cualitativos, los datos obtenidos a través de estas ocho entrevistas con mujeres inmigrantes no pueden generalizarse a la población de la cual se extrajo la muestra. Aunque todas las participantes compartieron su experiencia personal de aculturación, cada una posee una perspectiva distinta y única. La inclusión de un mayor número de mujeres hispanas puede proveer diferentes perspectivas las cuales enriquecerán el conocimiento del fenómeno de la aculturación.

\section{Sugerencias para futuras investigaciones y}

la práctica profesional

Los resultados de este estudio pueden ser usados para desarrollar programas de prevención así como servicios que cubran realmente las necesidades de las mujeres latinas viviendo en los Estados Uni- 
dos. El discurso de las participantes mostró lo importante que es tomar en cuenta las diferencias étnicas de la comunidad hispana para de esa manera adaptar los programas ya existentes y así responder a su idiosincrasia. De la misma manera, el contenido de este estudio puede ayudar al personal que trabaja con la comunidad hispana para proveer una atención más empática, respetando y comprendiendo los valores de las inmigrantes latinas a quienes están atendiendo. Finalmente, debido a la importancia que tiene el apoyo social y familiar que reciben los inmigrantes, es necesario que los programas de prevención y atención diseñados para las mujeres latinas potencialicen este recurso tan valioso para ellas.

Basado en los resultados de este estudio, un mayor número de líneas de investigación son necesarias para identificar los factores de riesgo que podrían incrementar el estrés, la ansiedad, los conflictos maritales y la depresión a la que se ven expuestos los inmigrantes, principalmente, durante los primeros días de residencia en los Estados Unidos. Por otra parte, la inclusión de métodos cuantitativos podría ayudar a determinar las relaciones entre las variables que emergieron de la narrativa de las inmigrantes participantes en este estudio, así como la magnitud de las mismas. A su vez, estudios de tipo longitudinal ayudarían a clarificar la cuestión de la direccionalidad de las variables asociadas al proceso de aculturación y a identificar los cambios más importantes que las inmigrantes van experimentando con el paso de los meses. Estos estudios deberían investigar el impacto de factores internos como lo son las características sociodemográficas y la personalidad de los 
inmigrantes, así como factores externos como lo son la discriminación, las características del área geográfica en donde viven, y las interacciones con los miembros de la comunidad norteamericana.

\section{Bibliografía}

Adelman, M. B. Cross-cultural adjustment: A theoretical perspective on social support. International Journal of Intercultural Relations, 12, 183-204, 1988.

AisENSTEIN, C. Inmigración y salud materno-infantil. Perinatología y Reproducción Humana, 15, 42-51, 2001.

ANDREAS, P. Border games: policing the Us-Mexico divide. New York, NY: Cornell University Press, 2000.

BACALlAO, M. L. y SMOKOWSKI, P. R. The costs of getting ahead: Mexican family system changes after immigration. Family Relations, 56, 52-66, 2007.

BERRY, J. W. Understanding and managing multiculturalism. Journal of Psychology and Developing Societies, 3, 17-49, 1991.

- Immigration, acculturation, and adaptation. Applied Psychology: An International Review, 46(1), 5-68, 1997.

- A psychology of immigration. Journal of Social Issues, 57(3), 615631, 2001.

—. "Acculturation strategies and adaptation", en J. Lansford (Ed.), Immigrant families in contemporary society (pp. 69-82). New York, NY: Guilford Publications, 2007. 
BONEVA, B. S. y FRIEZE, I. H. Toward a concept of a migrant personality. Journal of Social Issues, 57(3), 477-491, 2001.

Bourhis, R. Y.; MOISE, L. C.; PERREAult, S. y SEneCAL, S. Towards an interactive acculturation model: A social psychological approach. International Journal of Psychology, 32 (6), 369-386, 1997.

Canales, A. I. "Los migrantes latinoamericanos en Estados Unidos: Inserción laboral con exclusión social”, en Alejandro I. Canales Cerón (Ed.), Panorama actual de las migraciones en América Latina. México: Universidad de Guadalajara y Asociación Latinoamericana de Población, 2006.

CRESWELL, J. W. Qualitative inquiry and research design: Choosing among five approaches (2da. ed.). London: Sage Publications, 2007.

Daly, K. J. Qualitative methods for family studies and human development. London: Sage Publications, 2007.

Gamio, M. Mexican immigration to the United States. New York, NY: Arno Press and The New York Times, 1969.

George, U. y Ramkissoon, S. Race, gender, and class: Interlocking oppressions in the lives of South Asian women in Canada. Affilia Journal of Women and Social Work, 13(1), 102-120, 1998.

GonzÁlez, A. Mexican Americans and the U.S. economy: The Mexican American experience. Tucson, AZ: The University of Arizona Press, 2002.

HÄMÄLÄINEN, K. Unemployment, selective employment measures and inter-regional mobility of labor. Papers in Regional Science, 81, 423441, 2002. 
Hernández, M. y MCGOLDrick, M . "Migration and the family life cycle", en B. Carter y M. McGoldrick (Eds.), The expanded family life cycle: Individual, family, and social perspectives (3era. ed., pp. 169-184). Needham Heights, MA: Allyn \& Bacon, 1999.

Hill, C. E.; Thompson, B. J. y Williams, E. N. A guide to conducting consensual qualitative research. The counseling psychologist, 25, 517 572, 1997.

Hoefer, M.; Rytina, N. y BAKer, B. Estimates of the Unauthorized Immigrant Population Residing in the United States: January 2010. Washington, DC: Office of Immigration Statistics, Department of Homeland Security, 2011.

Hoffe, O. Breve historia ilustrada de la filosofía. Madrid: Península, 2003.

IÑIgUEZ, J. M. La inmigración de mujeres en la frontera sur de México y la participación femenina en los grupos Mara Salvatrucha 13 y Barrio 18. Revista de Economía y Relaciones Internacionales, 6(12), 65-80, 2008. Johnson, B. y Christensen, L. Educational research: Quantitative, qualitative and mixed approaches (3era. ed.). London: Sage Publications, 2008.

KIM, K. C. y HURH, W. M. Beyond assimilation and pluralism; syncretic sociocultural adaptation of Korean immigrants in the US. Ethnic and Racial Studies, 16(4), 696-713, 1993.

Kosic, A. Acculturation strategies, coping process and acculturative stress. Scandinavian Journal of Psychology, 45, 269-278, 2004.

MARTINS, V. y REID, D. New-immigrant women in urban Canada: Insights into occupation and sociocultural context. Occupational Therapy International, 14(4), 203-220, 2007. 
MAydell-steVens, E., MASGORET, A. y WARD, T. Problems of psychological and sociocultural adaptation among Russian-speaking immigrants in New Zealand. Social Policy Journal of New Zealand, 30, 178-198, 2007.

MelviLle, M. B. Mexican women adapt to migration. International Migration Review, 12 (2), 225-235, 1978.

Musetescu, R. International labor migration: Between human rights and political objectives. Romanian Economic and Business Review, 1, 34-42, 2006.

Neto, F. "Migration plans and their determinants among Portuguese adolescents", en J. W. Berry and R. C. Annis (eds.), Ethnic psychology: Research and practice with immigrants refugees, native peoples, ethnic groups and sojourners (pp. 308-314). Lisse: Swets \& Zeitlinger, 1988.

Ogbu. J. "Cultural models. identity and literacy", en J. Stigler, R. Shweder, and G. Herdt (Eds.), Cultural psychology: Essays on comparative human development (pp. 520-541). New York, NY: Cambridge University Press, 1990.

PASSEL, J. S. y D'vera COHN, S. W. Unauthorized immigrant population: National and state trends, 2010. Washington, DC: Pew Hispanic Center, 2011.

Patton, M. Q. How to use qualitative methods in evaluation. Newbury Park, CA: Sage Publications, 1987.

Ponterotto, J. G. Qualitative research in counseling psychology: A primer of research paradigms and philosophy of science. Journal of Counseling Psychology, 52(2), 126-136, 2005.

Redfield, R.; Linton, R. y HersKovits, M. Memorandum for the study of acculturation. American Anthropologist, 38, 149-152, 1936. 
RuIZ, J. J. y CANO, J. J. Manual de psicoterapia cognitiva. Madrid: Ubeda: R\&C Editores, 2002.

SÁnchez-huesca, R. y Arellanez-Hernández, J. L. Uso de drogas en migrantes mexicanos captados en ciudades de la frontera noroccidental México-Estados Unidos. Estudios Fronterizos, 12(23), 9-26, 2011.

SÁNCHEZ-QuinTANAR, C. Causas de los comportamientos migratorios desde la perspectiva de migrantes y no migrantes. Revista Mexicana de Psicología, 22(2),505-512, 2005.

SHIN, K. R. The lived experience of Korean immigrant women acculturating into the United States. Health Care for Women International, 20, 603617, 1999.

SkUZA, J. A. Humanizing the understanding of the acculturation experience with phenomenology. Human Study, 30, 447-465, 2007.

Solís-PÉrez, M. y Alonso-Meneses, G. Una caracterización de las mujeres en tránsito hacia Estados Unidos: 1993-2006. Papeles de población, 15(62), 253-283, 2009.

WARD, C. "Psychological theories of culture contact and their implications for intercultural training and interventions”, en D. Landis, J. M. Bennett, and M. J. Bennett (Eds.), Handbook of intercultural training (pp. 185-216). London: Sage Publications, 2004.

Yeh, C. J.; Arora, A. K.; Inose, M.; Okubo, Y.; Li. R. H. y Greene, P. The cultural adjustment and mental health of Japanese immigrant youth. Adolescence, 38, 481-496, 2004.

ZÚNigA, E., Leite, P. y NAVA, A. R. La nueva era de las migraciones: Características de la migración internacional en México. México: Conapo, 2003. 